\title{
Optimization of Excitation in FDTD Method and Corresponding Source Modeling
}

\author{
Bojan DIMITRIJEVIC, Bojana NIKOLIC, Slavoljub ALEKSIC, Nebojsa RAICEVIC
}

Faculty of Electronic Engineering, University of Nis, A. Medvedeva 14, 18000 Nis, Serbia

\{bojan.dimitrijevic, bojana.nikolic, slavoljub.aleksic, nebojsa.raicevic\}@elfak.ni.ac.rs

\begin{abstract}
Source and excitation modeling in FDTD formulation has a significant impact on the method performance and the required simulation time. Since the abrupt source introduction yields intensive numerical variations in whole computational domain, a generally accepted solution is to slowly introduce the source, using appropriate shaping functions in time. The main goal of the optimization presented in this paper is to find balance between two opposite demands: minimal required computation time and acceptable degradation of simulation performance. Reducing the time necessary for source activation and deactivation is an important issue, especially in design of microwave structures, when the simulation is intensively repeated in the process of device parameter optimization. Here proposed optimized source models are realized and tested within an own developed FDTD simulation environment.
\end{abstract}

\section{Keywords}

Finite difference time domain, source modeling, excitation, optimization

\section{Introduction}

The finite difference time domain (FDTD) method currently draws significant scientific attention as one of the most efficient methods for analysis and characterization of wide range of electromagnetic problems [1]. The proper excitation and source modeling in the FDTD computational domain is especially important issue in every application of FDTD simulation.

Introduction of discrete internal sources is usually done by applying either hard source or soft source excitation. The hard source excitation is consisted in assigning specific value of certain electric (or magnetic) field component at a single or several grid points in every time step through an appropriate time function [2]. The soft source excitation is introduced by adding the appropriate time function to the field value obtained in regular update equation [1].

Although it is not a physical reality, the plane wave excitation has enormously large significance in many theo- retical and analytical considerations. For this reason it is very important to introduce the same excitation in simulation environments and to enable the comparative analysis of the results. The necessity of plane-wave source arose originally with the first FDTD modeling in the field of defense and bioelectromagnetics [1]. Considering scattering problems, where the particular structure of interest is far away from the radiation source and the incident wave can be considered as a plane wave, Yee was the first to introduce the initial-condition approach [3]. However, today mainly accepted approach for plane-wave excitation is total-field/ scattered-field (TF/SF) formulation [4], [5]. The TF/SF technique showed very good performance in FDTD modeling of long-duration pulsed or continuous wave excitation and it is widely used in guided-wave simulations [1]. The TF/SF technique has been extensively studied in the literature and many modifications and improvements of this basic method can be found [6-9].

However, another way of plane wave excitation modeling includes adding or assigning of an electric (or magnetic) field value at specific positions in one plane, unlike the commonly used TF/SF technique, where corrections are made in both electric and magnetic field components (displaced in time and space for a half time step) on the boundary surface. The advantage of this direct approach is its simplicity. Its main difficulty is, however, the existence of wave propagation in undesirable direction. But since very effective boundary conditions like the convolutional perfectly matched layer (CPML) [10] are available, this is no longer an obstacle to its application. The considerations regarding this approach can be found in [11].

The excitation modeling in FDTD formulation significantly affects the simulation performance. A sudden excitation of the domain causes undesirable numerical variations in whole computational domain. This problem is usually resolved by slow introduction of the source excitation, using the appropriate shaping functions in time. A number of time functions for slow introduction of source excitation are available in the literature [12]. However, a gradual raise of the excitation signal is time consuming and can be a significant difficulty in applications where the intensive repetition of simulations is required. For this reason, a certain compromise between the required time and satisfactory simulation performance must be achieved. 
The optimization presented in this paper is conducted in order to minimize the propagation of the undesirable energy through the computational domain. As a result of the optimization process, very simple and closed-form optimal function is obtained. Numerically obtained optimal shaping function is compared with the solutions that can be found in the literature and some general remarks are derived.

\section{Source Modeling}

In hard source modeling, instead of calculating field value using FDTD update equations, the field value in the specific grid points is assigned using a time function. If the source is at position $(i, j, k)$, it is then

$$
E_{v(i, j, k)}^{n}=E_{\text {source }}^{n}, \mathrm{v}=(x, y, z) .
$$

In the same manner as in the case of the point source, the plane wave source can be also modeled as a hard source, applying (1) on a group of points belonging to a specific surface or even volume. Regardless of the case, hard source model is equivalent to the ideal voltage source and for this reason acts like an electric wall, causing reflections of any wave arrived to the source location [2], [13]. This means that such a source, more specifically in the case of plane wave excitation separates the computational domain in two independent regions without mutual interaction. Hard source modeling is commonly applied in excitation of guided-wave structures. In [14] the analytical solution for the FDTD hard source has been derived, so its application is broaden to the validation of FDTD codes or FDTD schemes.

One possible solution to the reflective behavior of the hard sources is to combine it with the regular FDTD update equations [1]. Namely, the hard source can be turned on only while there is a signal excitation. The simulation setup in that case should provide that the signal excitation ends before any scattered or reflected wave returns to the source position. After this period, the usual FDTD update of the field components can be applied. The main difficulty of this approach is that it can't be used for continuous excitation.

In soft source modeling, source excitation is added to the value obtained in applied FDTD update equations. If the source is at position $(i, j, k)$, it is then

$$
E_{\mathrm{v}(i, j, k)}^{n}=E_{\mathrm{v}(i, j, k) \text { FDTDupdate }}^{n}+E_{v_{\text {source }}}^{n}, \mathrm{v}=(x, y, z)
$$

The main advantage of the soft source is the fact that it is transparent to the incoming waves and it allows the different incident fields to interact [1], [15]. For soft source modeling of the plane wave it can be either TF/SF method or method of direct adding/assigning of electric (magnetic) field value [11] applied. When point soft source modeling is concerned, the additional source component is usually introduced through the current density $J_{v(i, j, k)}^{t+1 / 2}$, which is defined at the same spatial position as the resulting electric field component but the time step is the same as the one of the magnetic field. If the source is at position $(i, j, k)$, it is then

$$
\begin{gathered}
\frac{\Delta E_{v(i, j, k)}^{t+1}}{\Delta t}=\frac{1}{\varepsilon_{0}} \frac{\Delta H_{w(i, j, k)}^{t+1 / 2}}{\Delta u}-\frac{1}{\varepsilon_{0}} \frac{\Delta H_{u(i, j, k)}^{t+1 / 2}}{\Delta w}-\frac{1}{\varepsilon_{0}} J_{v(i, j, k)}^{t+1 / 2} \\
v=(x, y, z), u=(z, x, y), w=(y, z, x), \\
i=\left\{0, \ldots, N_{x}-1\right\}, j=\left\{0, \ldots, N_{y}-1\right\}, k=\left\{0, \ldots, N_{z}-1\right\}, \\
n=\left\{0, \ldots, N_{t}-1\right\}
\end{gathered}
$$

where $\Delta t$ is the time step, $\Delta x, \Delta y$ and $\Delta z$ are spatial steps along $x, y$ and $z$ axis, respectively. Total numbers of elementary cells along $x, y$ and $z$ axis are denoted by $N_{x}, N_{y}$ and $N_{z}$, respectively. Total number of elementary time steps is denoted by $N_{t}$. The electric magnetic permittivity in vacuum is denoted by $\varepsilon_{0}$.

Since the FDTD cell is usually much shorter than one-tenth of the main wavelengths of interest, physically the soft source current acts as a Hertzian dipole antenna [14]. The point soft source physically corresponds to real current (or voltage) source.

One of the main difficulties regarding the soft source modeling is constant deposit of charges and generation of the charge-associated fields [16], [1]. This can be circumvented by using matched voltage or current sources. By applying the source resistance $R_{S}$, voltage source at position $(i, j, k)$ can be introduced in update equation of the corresponding field component as

$$
\begin{gathered}
E_{v(i, j, k)}^{t+1}=\frac{1-b}{1+b} E_{v(i, j, k)}^{t}+ \\
+\frac{\Delta t / \varepsilon_{0}}{1+b}\left(\frac{\Delta H_{w(i, j, k)}^{t+1 / 2}}{\Delta u}-\frac{\Delta H_{u(i, j, k)}^{t+1 / 2}}{\Delta w}-\frac{V_{S v(i, j, k)}^{t+1 / 2}}{R_{S} \Delta u \Delta w}\right) \\
b=\frac{\sigma \Delta t}{2 \varepsilon_{0}}+\frac{\Delta t \Delta v}{2 R_{S} \varepsilon_{0} \Delta u \Delta w}
\end{gathered}
$$

where $\sigma$ is the electric conductivity.

It is shown in [14] that the fields radiated by hard and soft source models are identical and the relation that connects excitation $E_{v \text { source }}^{n}$ from (1) and $J_{v(i, j, k)}^{t+1 / 2}$ from (2) can be expressed as

$$
\begin{gathered}
E_{v \text { source }}^{n}=-\frac{1}{3 \varepsilon_{0}} \int_{-\infty}^{t_{n}} J_{S}(t) d t, \\
J_{S}\left(t_{n+1 / 2}\right)=J_{v(i, j, k)}^{n+1 / 2} .
\end{gathered}
$$

\section{Excitation Modeling}

A proper excitation of the FDTD computational domain is important in every FDTD application. The abrupt 
source introduction yields intensive numerical variations at high frequencies which propagate through the whole computational domain. A generally accepted solution is to slowly introduce the source. In order to fulfill this, it is necessary for the excitation function to satisfy following conditions: at zero time step the excitation function value must be zero (if fields are initiated at zero) and the excitation function must be smooth. The most commonly used time functions for slow introducing of pulsed source excitation available in the literature are given in Tab. 1.

\begin{tabular}{|c|c|}
\hline $\begin{array}{l}\text { Broad band Gaussian } \\
\text { with DC component }\end{array}$ & $f(t)=\exp \left(-\left(t-t_{0}\right)^{2} / t_{w}^{2}\right), 0<t<2 t_{0}$ \\
\hline $\begin{array}{l}\text { Broad band Gaussian } \\
\text { without DC component } \\
\text { - Gaussian derivative }\end{array}$ & $f(t)=\frac{-2}{t_{w}}\left(t-t_{0}\right) \exp \left(-\left(t-t_{0}\right)^{2} / t_{w}^{2}\right)$ \\
\hline $\begin{array}{l}\text { Blackman - Harris } \\
\text { window }\end{array}$ & $\begin{array}{l}b(t)=\sum_{n=0}^{3} a_{n} \cos \left(\frac{2 \pi n t}{T}\right), \quad 0<t<T \\
a=\left[\begin{array}{c}0.353222222 \\
-0.488 \\
0.145 \\
-0.010222222\end{array}\right], T=\frac{1.55}{f_{b w}} \\
f_{b w}-\text { half bandwidth of the pulse }\end{array}$ \\
\hline $\begin{array}{l}\text { Differentiated } \\
\text { Blackman - Harris } \\
\text { window }\end{array}$ & $d_{b}(t)=-\sum_{n=0}^{3} a_{n} n \sin \left(\frac{2 \pi n t}{T}\right), 0<t<T$ \\
\hline Raised cosine [17] & $\begin{array}{l}z(t)=0.5\left(1-\cos \left(\frac{2 \pi t}{T}\right)\right), 0<t<T_{r} / 2 \\
T_{r} \text { - period of ramped cosine }\left(T_{r} \approx 3 T\right)\end{array}$ \\
\hline
\end{tabular}

Tab. 1. Time functions used for pulsed excitation [12].

The raised cosine [17] is considered to be the most suitable for excitation of FDTD domain and it is the preferred choice, especially compared to linear and exponential ramps [15]. However, the presented excitation functions (Tab. 1) are not designed to meet the specific requirements of FDTD formulation. In order to get a better insight in FDTD nature, we shell start from the case of $z$-polarized plane wave, propagating along $y$ axis. Ampere's and Faraday's law in that case have the form

$$
\begin{gathered}
\frac{\partial H_{x}}{\partial t}=\frac{1}{\mu_{0}} \frac{\partial E_{z}}{\partial y}, \\
\frac{\partial E_{z}}{\partial t}=\frac{1}{\varepsilon_{0}} \frac{\partial H_{x}}{\partial y}-\frac{J_{S}}{\varepsilon_{0}} .
\end{gathered}
$$

If one differentiates (1) over space variable $y$ and (2) over time $t$, it yields

$$
\begin{gathered}
\frac{\partial^{2} H_{x}}{\partial y \partial t}=\frac{1}{\mu_{0}} \frac{\partial^{2} E_{z}}{\partial y^{2}}, \\
\frac{\partial^{2} E_{z}}{\partial t^{2}}=\frac{1}{\varepsilon_{0}} \frac{\partial^{2} H_{x}}{\partial t \partial y}-\frac{1}{\varepsilon_{0}} \frac{\partial J_{S}}{\partial t} .
\end{gathered}
$$

Substituting (8) in (9), it is obtained

$$
\frac{\partial^{2} E_{z}}{\partial t^{2}}=\frac{1}{\varepsilon_{0}} \frac{1}{\mu_{0}} \frac{\partial^{2} E_{z}}{\partial y^{2}}-\frac{1}{\varepsilon_{0}} \frac{\partial J_{S}}{\partial t} .
$$

Equation (10) indicates that the second time derivative of the excitation function should be the one to investigate, since it causes the propagation through the computational domain.

\section{Numerical Optimization of FDTD Domain Excitation Function}

In order to minimize the propagation of the undesirable energy through the FDTD computational domain that consequently appears during its excitation, the minimization of the second time derivative of the excitation function should be performed.

Without loss of generality, the excitation function will be analyzed in its normalized form. Considering (10) and [17], in order to have the desirable properties, the excitation function $f(x)$ should satisfy the following criteria

1. $f(0)=0$ and $f(1)=1$;

2. $f(x)$ is an odd function with respect to the point $(1 / 2$, $1 / 2$ ) in Cartesian coordinate system;

3. the first derivative of $f(x)$ is continuous function and $f^{\prime}(0)=0$ (in order to avoid large values in the second derivative).

Two possible solutions are considered.

\subsection{Polynomial Optimization}

For the purpose of optimization, the excitation function will be presented as a linear combination of basic functions which satisfy conditions 1, 2 and 3. Considered basic functions are in polynomial form and given as

$$
f_{n}(x)= \begin{cases}0, & x<0 \\ 2^{n-1} x^{n}, & 0 \leq x \leq \frac{1}{2}, n=2,3, \ldots \\ 1-2^{n-1}(1-x)^{n}, & \frac{1}{2} \leq x \leq 1 \\ 1, & x>1\end{cases}
$$

If functions $f_{n}(x)$ fulfill conditions 1,2 and 3 , then their linear combination

$$
f(x)=C_{2} f_{2}(x)+C_{3} f_{3}(x)+\ldots
$$

also fulfills the same condition 3. However, there is an additional requirement for $f(x)$, in order to satisfy the conditions 1 and 2

$$
\sum_{i=2}^{+\infty} C_{1}=1
$$

Applying numerical iterative minimization of the mean square value of the second derivative $\partial^{2} f(x) / \partial x^{2}$ $\left(\min _{C_{i}, i=2,3,4, \ldots} \overline{f^{\prime \prime 2}(x)}\right)$, it is obtained 


$$
C_{2}=\frac{3}{2}, C_{3}=\frac{1}{2}, C_{4}=C_{5}=C_{6}=\ldots=0 .
$$

Thus, the optimal excitation function has the form

$$
f_{\text {opt }}(x)= \begin{cases}0, & x<0 \\ 3 x^{2}-2 x^{3}, & 0 \leq x \leq 1 \\ 1, & x>1\end{cases}
$$

\subsection{Trigonometric Optimization}

If the basic functions in series expansion of the excitation function are

$$
f_{m}^{(M)}(x)= \begin{cases}1, & x<0 \\ \cos (2 m+1) \pi x, & 0 \leq x \leq 1 \\ -1, & x>1\end{cases}
$$

the excitation function is then

$$
f^{(M)}(x)=\frac{1}{2}-\sum_{m=0}^{M} a_{2 m+1} f_{2 m+1}^{(M)}(x)
$$

where $a_{2 m+1}$ are series coefficients, which should fulfill the criterion

$$
\sum_{m=0}^{M} a_{2 m+1}=\frac{1}{2}
$$

Applying numerical iterative minimization of the mean square value of the second derivative $\partial^{2} f^{(M)}(x) / \partial x^{2}$ in this case, a function with expansion coefficients presented in Tab. 2 for different $M$ values is obtained. The mean square values of the second derivative of function $f^{(M)}(x)$ are also given.

\section{Optimization Results}

In Fig. 1 excitation function obtained in the optimization process using polynomial basic functions (denoted as $\mathrm{Opt}$ ), as well as the ones using trigonometric basic functions for different values of $M$ are presented. The curve that corresponds to the value $M=0$ is actually the excitation function that is widely used in the literature and known as raised cosine.

In Fig. 2 one can observe the second derivatives of the functions from Fig. 1. It can be seen from Fig. 2 that the second derivative of the excitation function $M=0$ (raised cosine) significantly deviates from the second derivative of the optimal function obtained using polynomial expansion. It can be also observed that with the increase of $M$ the second derivative of the function with trigonometric expansion converges to the one of the optimal function with polynomial expansion. This confirms that the same optimal result is obtained regardless of the applied type of basic functions in optimization process. Since the solution

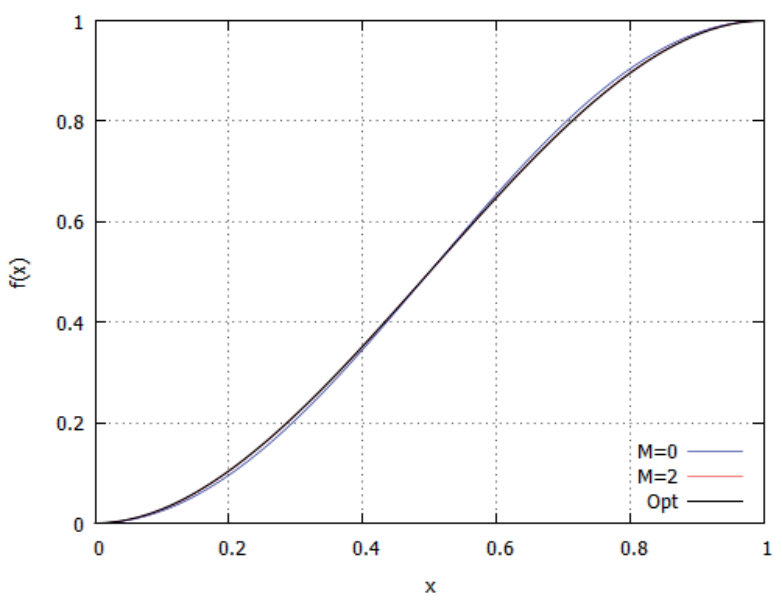

Fig. 1. The proposed optimized excitation functions.

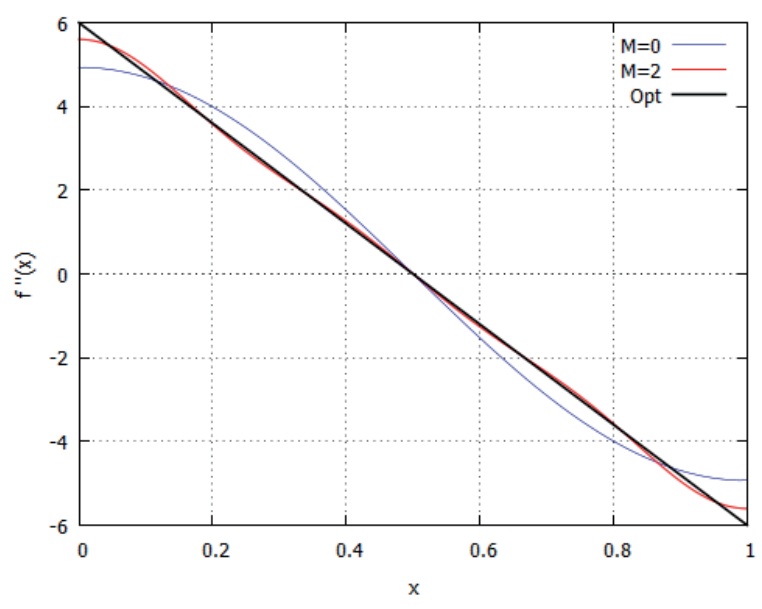

Fig. 2. Second derivative of the proposed optimized excitation functions.

obtained in polynomial form is simple and in a closed form, we propose function (15) as the excitation function for FDTD domain.

Optimality of the obtained function shape is verified in an own developed FDTD simulation environment [18].

\section{Optimal Excitation Signals}

Using the obtained optimal excitation function (Fig. 3), two pulsed signals are proposed for efficient excitation of FDTD domain. The total pulsed signal retains the optimal properties only if it is formed using the proposed optimal function as segments that are appropriately symmetrically extended or scaled in time and amplitude. Thus, the proposed pulsed function has the form

$$
f_{p}(x)=\left\{\begin{array}{lc}
0, & x<0 \\
3 x^{2}-2 x^{3}, & 0 \leq x \leq 1 \\
3(2-x)^{2}-2(2-x)^{3}, & 1 \leq x \leq 2 \\
0, & x>2
\end{array}\right.
$$




\begin{tabular}{|c|c|c|c|c|c|c|c|}
\hline$M$ & $\overline{f^{\prime \prime 2}(x)}$ & $a_{1}$ & $a_{3}$ & $a_{5}$ & $a_{7}$ & $a_{9}$ & $\cdots$ \\
\hline 0 & 12.1761 & 0.5 & - & - & - & - & - \\
\hline 1 & 12.0276 & 0.493902 & 0.00609756 & - & - & - & - \\
\hline 2 & 12.0086 & 0.493123 & 0.00608793 & $7.88996 \times 10^{-4}$ & - & - & - \\
\hline 3 & 12.0037 & 0.492920 & 0.00608543 & $7.88672 \times 10^{-4}$ & $2.05298 \times 10^{-4}$ & - & - \\
\hline 4 & 12.0019 & 0.492846 & 0.00608452 & $7.88554 \times 10^{-4}$ & $2.05267 \times 10^{-4}$ & $7.51175 \times 10^{-5}$ & - \\
\hline$\rightarrow \infty$ & 12.0000 & 0.492767 & 0.00608354 & $7.88427 \times 10^{-4}$ & $2.05234 \times 10^{-4}$ & $7.51054 \times 10^{-5}$ & $\ldots$ \\
\hline
\end{tabular}

Tab. 2. Expansion coefficients in $f^{(M)}(x)$.

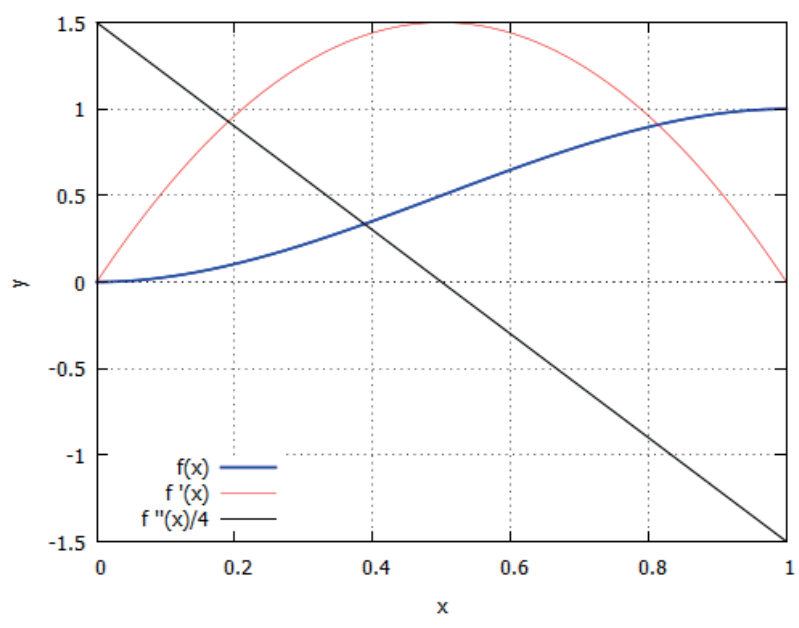

Fig. 3. Proposed optimal excitation function and its first and second derivative.

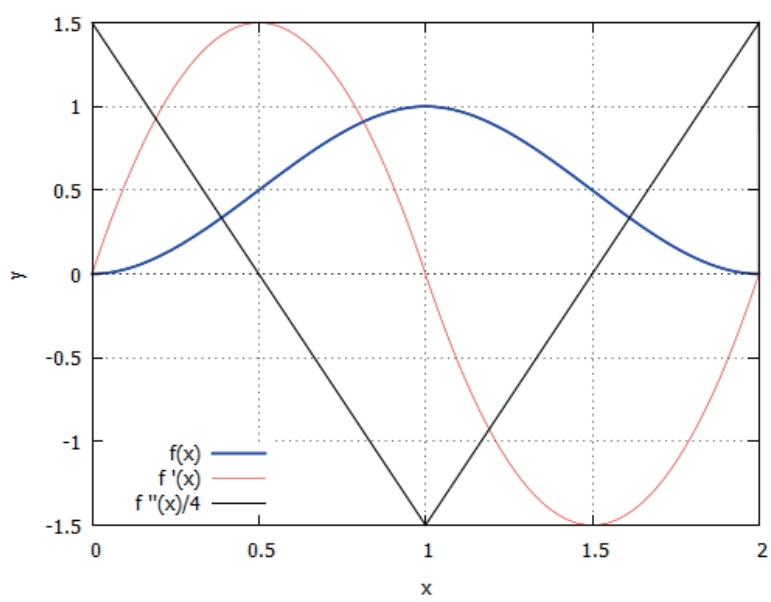

Fig. 4. Proposed optimal pulsed excitation function with DC component and its first and second derivative.

Function (19) along with its first and second derivative is presented in Fig. 4.

In case the pulsed signal with no DC component is required, it should be also obtained as symmetrically extended or adequately scaled optimal function (15). The pulsed signal with no DC component shouldn't be formed as the first derivative of the optimal pulsed function (Fig. 4), because in that case the resulting function would change its nature and wouldn't have optimal properties any more. Thus, we propose the pulsed function with no DC component in the form

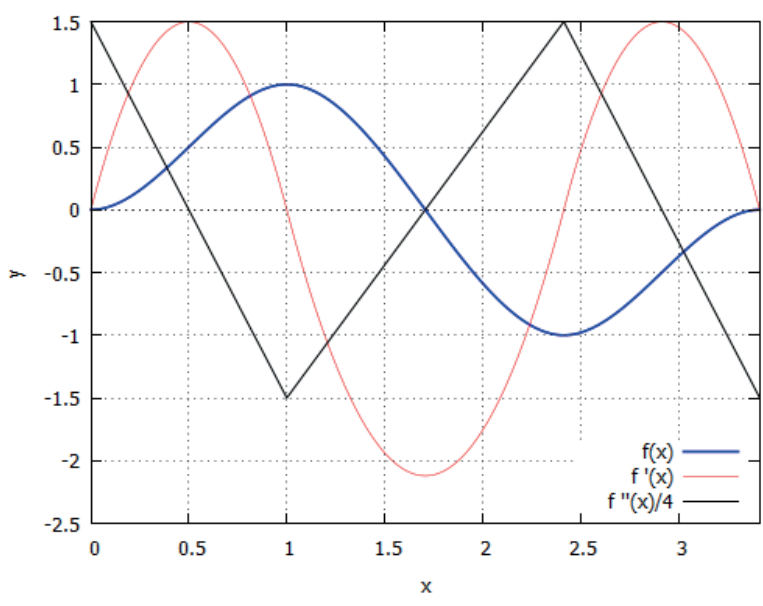

Fig. 5. Proposed optimal pulsed excitation function without DC component and its first and second derivative.

$$
\begin{aligned}
& f_{d}(x)= \\
& =\left\{\begin{array}{lc}
0, & x<0 \\
3 x^{2}-2 x^{3}, & 0 \leq x \leq 1 \\
3(1+\sqrt{2}-x)^{2}-\sqrt{2}(1+\sqrt{2}-x)^{3}-1, & 1 \leq x \leq 1+\sqrt{2} \\
-3(2+\sqrt{2}-x)^{2}+\sqrt{2}(2+\sqrt{2}-x)^{3}, & 1+\sqrt{2} \leq x \leq 2+\sqrt{2} \\
0, & x>2+\sqrt{2}
\end{array}\right.
\end{aligned}
$$

It is interesting to mention that in (20) in the function segment $1 \leq x \leq 1+\sqrt{2}$ the required transition is from 1 to -1 , thus the optimal function (15) should be scaled by $\sqrt{2}$ over time and by 2 over amplitude in order to keep its optimal characteristics. Function (20), along with its first and second derivative, is presented in Fig. 5.

\section{Conclusion}

The first part of this paper contains the overview of the relevant principles in source modeling in FDTD, with special focus on differences between hard and soft sources and on different source geometry (plane wave sources and point sources). The second part of the paper is dedicated to the source optimization, more specifically to the optimization of the excitation time function, which has a significant influence on the behavior of the generator, regardless of its type. Generally accepted and the most frequently used 
excitation functions are listed. However, neither of them is designed primarily for FDTD application, taking into account specificities of FDTD method.

The optimization problem in this work is defined in order to minimize the propagation of the undesirable energy through the computational domain. This is accomplished by minimizing the mean square value of the second time derivative of the excitation function. As a result of the optimization process, very simple and closed-form optimal function is obtained.

In addition, two functions for pulsed excitation of FDTD domain, with and without DC component, are proposed.

Optimality of the obtained function shape is verified in the own developed FDTD simulation environment.

\section{References}

[1] TAFlove, A., HAGNESS, S. B. Computational Electrodynamics: The Finite-Difference Time-Domain. $3^{\text {rd }}$ ed. Boston: Artech House, 2005.

[2] BUECHLER, D. N., ROPER, D., DURNEY, C. H. CHRISTENSEN, D. A. Modeling sources in the FDTD formulation and their use in quantifying source and boundary condition errors. IEEE Transactions on Microwave Theory and Techniques, 1995, vol. 43, p. 810-814. DOI: $10.1109 / 22.375228$

[3] YEE, K. S. Numerical solution of initial boundary value problems involving Maxwell's equations in isotropic media. IEEE Transactions on Antennas and Propagation, 1966, vol. 14, no. 3, p. 302-307. DOI: 10.1109/TAP.1966.1138693

[4] MEREWETHER, D. E., FISHER, R., SMITH, F. W. On implementing a numeric Huygen's source scheme in a finite difference program to illuminate scattering bodies. IEEE Transactions Nuclear Science, 1980, vol. 27, no. 6, p. 1829-1833. DOI: $10.1109 /$ TNS.1980.4331114

[5] UMASHANKAR, K. R., TAFLOVE, A. A novel method to analyze electromagnetic scattering of complex objects. IEEE Transactions on Electromagnetic Compatibility, 1982, vol. 24, no. 4, p. 397-405. DOI: 10.1109/TEMC.1982.304054

[6] WATTS, M. E., DIAZ, R. E. Perfect plane-wave injection into a finite FDTD domain through teleportation of fields. Electromagnetics, 2003, vol. 23, p. 187-201. DOI: $10.1080 / 02726340390159504$

[7] OGUZ, U., GUREL, L., ARIKAN, O. An efficient and accurate technique for the incident-wave excitations in the FDTD method. IEEE Microwave and Guided Wave Letters, 1998, vol. 46, no. 6 , p. 869-882. DOI: $10.1109 / 22.681215$

[8] TAN, T., POTTER, M. Optimized analytic field propagator $(\mathrm{O}$ AFP) for plane wave injection in FDTD simulations. IEEE Transactions on Antennas and Propagation, 2010, vol. 58, no. 3, p. 824-831. DOI: 10.1109/TAP.2009.2039310

[9] GUIFFAUT, C., MAHDJOUBI, K. A perfect wideband plane wave injector for FDTD method. In Proceedings of the IEEE International Symposium on Antennas and Propagation. Salt Lake City (UT, USA), 2000, vol. 1, p. 236-239. DOI: 10.1109/APS.2000.873752

[10] RODEN, J. A., GEDNEY, S. D. Convolution PML (CPML): An efficient FDTD implementation of the CFS-PML for arbitrary media. Microwave and Optical Technology Letters, 2000, vol. 27, no. 5 , p. 334-339.

[11] MANSOURABADI, M., POURKAZEMI, A. FDTD hard source and soft source reviews and modifications. Progress In Electromagnetics Research C, 2008, vol. 3, p. 143-160. DOI: 10.2528/PIERC08032302

[12] GEDNEY, S. D. Introduction to the Finite-Difference TimeDomain (FDTD) Method for Electromagnetics. Course text, Morgan and Claypool Publishing, 2011. [Online] Cited 2015-0303. Available at: http://www.engr.uky.edu/ gedney/courses/ee624.

[13] INAN, I. M., MARSHALL, R. A. Numerical Electromagnetics The FDTD Method. Cambridge: Cambridge University Press, 2011.

[14] COSTEN, F., BERENGER, J.-P., BROWN, A. Comparison of FDTD hard source with FDTD soft source and accuracy assessment in Debye media. IEEE Transactions on Antennas and Propagation, 2009, vol. 57, no. 7, p. 2014-2022. DOI: 10.1109/TAP.2009.2021882

[15] KALIALAKIS, C. Finite Difference Time Domain Analysis of Microstrip Antenna-Circuit Modules. PhD Thesis. School of Electronic and Electrical Engineering, The University of Birmingham, July 1999.

[16] WAGNER, C. L., SCHNEIDER, J. B. Divergent fields, charge and capacitance in FDTD simulations. IEEE Transactions on Microwave Theory and Techniques, 1998, vol. 46, no. 12, p. 2131-2136. DOI: $10.1109 / 22.739294$

[17] ROPER, D. H., BAIRD, J. M. Analysis of overmoded waveguides using the finite difference time domain method. In Digest Proceedings of the IEEE Microwave Theory and Techniques Society Symposium. Albuquerque (USA), 1992, p. 401-404. DOI: 10.1109/MWSYM.1992.187997

[18] NIKOLIC, B., DIMITRIJEVIC, B., RAICEVIC, N, ALEKSIC, S. Implementation of FDTD based simulation environment. Facta Universitatis. Ser.: Electronics and Energetics. 2013, vol. 26, p. 121-132. DOI: 10.2298 /FUEE1302121N

\section{About the Authors ...}

Bojan DIMITRIJEVIĆ was born in Leskovac, Serbia, in 1972. He received the B.E.E., M.Sc., and Ph.D. degrees from the University of Niš in 1998, 2002, and 2006, respectively. His research interests include digital signal processing in telecommunications with special focus on interference suppression, adaptive filtering and synchronization, numerical methods in electromagnetics with special focus on FDTD method and signal, material and component modeling.

Bojana NIKOLIĆ was born in Niš, Serbia in 1982. She received the Dipl. - Ing. and Ph.D. degrees in Telecommunications from the Faculty of Electronic Engineering in Niš in 2007 and 2012, respectively. Her research interests include FDTD numerical modeling in electromagnetics and wireless communications.

Slavoljub ALEKSIĆ was born in Berčinac, Serbia in 1951. He received Dipl. - Ing., M. Sc. and Ph.D. degrees in Theoretical Electrical Engineering from the Faculty of Electronic Engineering, University of Niš, Serbia in 1975, 1979 and 1997, respectively. His researching areas are: electromagnetic field theory, numerical methods in elec- 
tromagnetics, lightning protection systems, low-frequency EM fields, microstrip transmission lines with isotropic, anisotropic and bianisotropic media, cable joints and cable terminations, permanent magnets analysis, power lines.

Nebojša RAIČEVIĆ was born in Niš, Serbia in 1965. He received his the Dipl. - Ing., M.Sc. and Ph.D. degrees from the Faculty of Electronic Engineering of Niš, Serbia, in
1989, 1998 and 2010, respectively. His research interests include: cable terminations and joints, numerical methods for electromagnetic problems solving, microstrip transmission lines with isotropic, anisotropic and bianisotropic media, analysis of metamaterial structures, electromagnetic compatibility, nonlinear electrostatic problems, magnetic field calculation of coils and permanent magnets. 